\title{
Erratum to: Capillary and van der Waals forces between uncharged colloidal particles linked by a liquid bridge
}

\author{
David Megias-Alguacil $\cdot$ Ludwig J. Gauckler
}

Received: 11 June 2010 / Accepted: 1 July 2010 /Published online: 2 September 2010

(C) Springer-Verlag 2010

\section{Erratum to: Colloid Polym Sci \\ DOI 10.1007/s00396-009-2106-0}

In a recent paper published in Colloid and Polymer Science [1] dealing with the comparison between the capillary forces and the van der Waals ones established between colloidal particles linked by concave liquid bridges, unfortunately there, there is a sign error in Eq. 8, which describes the capillary force between a pair of particles. The correct equation should read, as pointed out by Bakken et al. [2]:

$$
\begin{aligned}
F_{c a p}= & -2 \pi \gamma R \sin \alpha \sin (\alpha+\theta) \\
& +\pi \gamma R^{2} \sin ^{2} \alpha\left(\frac{1}{L}-\frac{1}{\rho}\right)
\end{aligned}
$$

where $\gamma$ is the surface tension of the liquid of the bridge, $R$ is the solid particle's radius, $\alpha$ is the half-filling angle, $\theta$ is the wetting angle and $\rho$ and $L$ are the principal radii of the liquid meniscus [1].

This correction does not affect the method of determining the liquid meniscus shape neither the limiting inter-particle distances of the concave liquid bridges, although Eq. 1 alters the published [1] shape of the capillary force vs. inter-particle distance, $H$. With the correct equation, repulsive capillary forces will only persist for wetting angles, $\theta$, lower than $\approx 20^{\circ}$ in a rather limited liquid volume range, $V_{\text {rel }}<0.023$, and just for the longest possible distances between particles, practically

The online version of the original article can be found at http://dx.doi. org/10.1007/s00396-009-2106-0.

D. Megias-Alguacil $(\bowtie) \cdot$ L. J. Gauckler

Department of Materials, Non-Metallic Inorganic Materials,

ETH-Zürich,

8093 Zürich, Switzerland

e-mail: david.megias@mat.ethz.ch immediately before the bridge's rupture, as displayed in Fig. 1.

When comparing the corrected capillary forces with the van der Waals ones, Fig. 2, qualitatively, similar results than already published [1] are found, but now the distances at which the ratio $F_{v d W} / F_{c a p}$ diverges are displaced to longer inter-particle separations.

With the correct equation for the capillary force, the induced deep minimum on the total force at inter-particles distances close to zero is still persisting. Since the van der Waals force becomes very small when the particles separate and $H_{0}$ (distances at which $F_{c a p}=0$ ) are now found at larger distances than before [1], as displayed in Fig. 1, the effect of $F_{v d W}$ on $H_{0}$ is rather small. It is only at extremely low liquid contents $\left(V_{r e l}<10^{-5}\right)$ and wetting angles $\theta \leq 20^{\circ}$ where

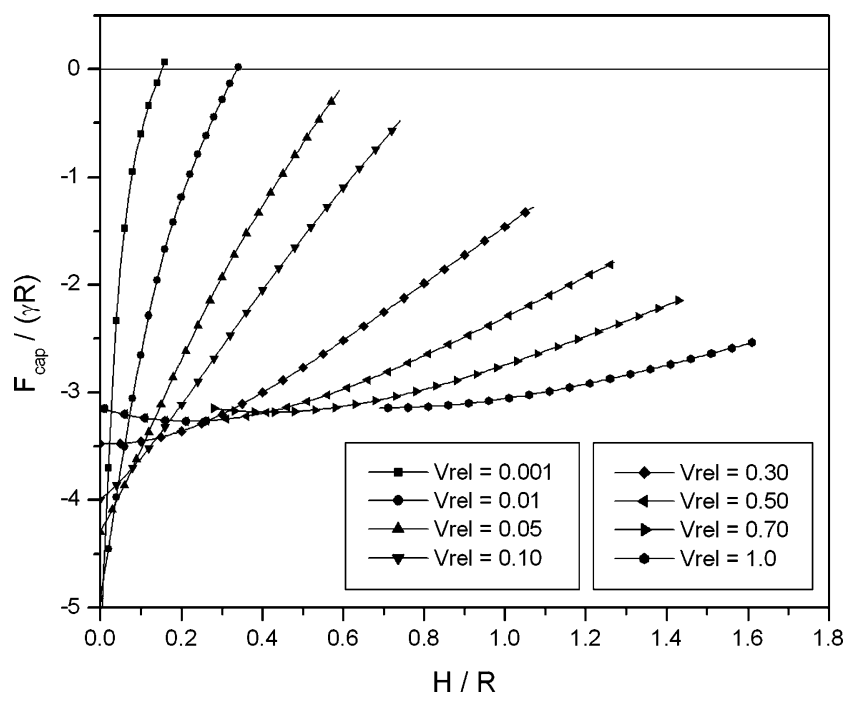

Fig. 1 Scaled capillary force, $F_{c a p} /(\gamma R)$, as a function of the dimensionless inter-particle distance, $H / R$, for different liquid contents when $\theta=0^{\circ}$, according to Eq. 1 


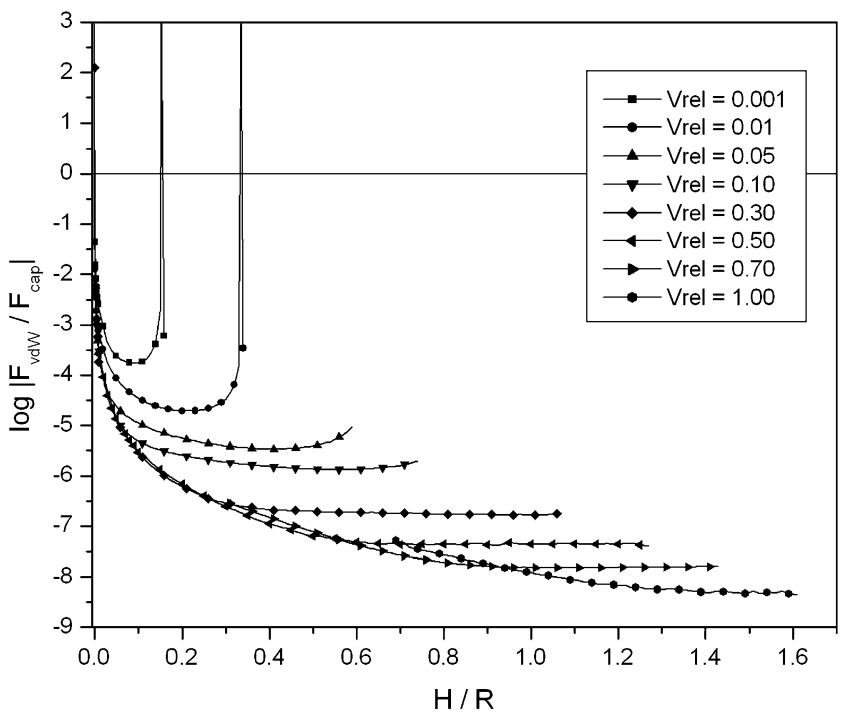

Fig. 2 Ratio $F_{v d W} / F_{c a p}$ as a function of the dimensionless interparticles distance, for several relative volume of meniscus liquid, $V_{\text {rel }}$, being $\theta=0^{\circ}$ and $A_{d r y}=10 A_{\text {wet }}$

the impact of the van der Waals force is noticeable, shifting the distances at which $F_{\text {tot }}=F_{c a p}+F_{v d W}=0$ to slightly larger values, as shown in Fig. 3. The van der Waals force, $F_{v d W}$, contribution to $H_{0}$ is at most $20 \%$, value reached in the limit of vanishing liquid content and $\theta=20^{\circ}$. For decreasing angles, the impact of $F_{v d W}$ on $H_{0}$ is practically negligible.

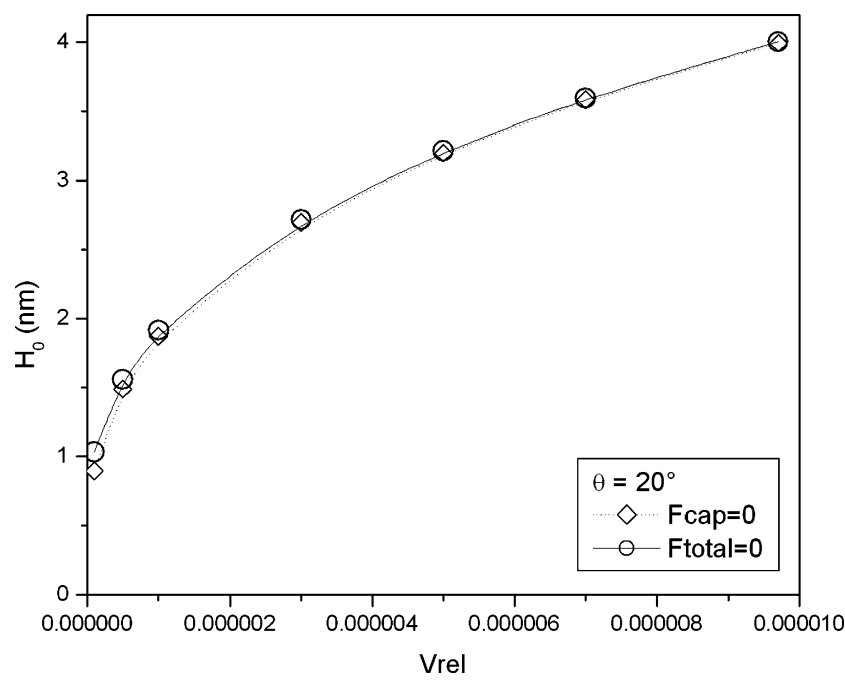

Fig. 3 Inter-particles distances, $H_{0}$, at which $F_{c a p}$ and $F_{t o t}$ are zero, as a function of the relative volume of meniscus liquid, $V_{\text {rel }}$, for wetting angles $\theta=20^{\circ}$

\section{References}

1. Megias-Alguacil D, Gauckler LJ (2010) Capillary and van der Waals forces between uncharged colloidal particles linked by a liquid bridge. Colloid Polym Sci 288:133-139

2. Bakken M, McCulfor J, Anklam MR (2010) Letter to the Editor. AIChE J 56:2489-2490 